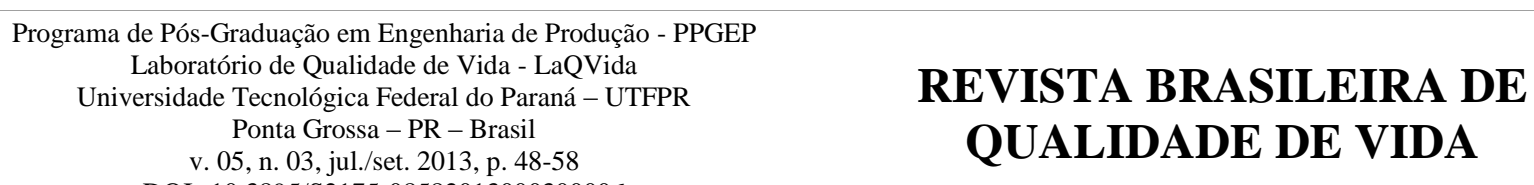

DOI: $10.3895 / \mathrm{S} 2175-08582013000300006$

\title{
Aspectos alimentares em idosos com diferentes condições dentárias
}

\author{
Diet Aspects in the elderly with different dental conditions
}

\author{
Karen Ullmann \\ Universidade Tuiuti do Paraná - TUIUTI - Curitiba- Brasil \\ kaullmann@gmail.com \\ Jair Mendes Marques \\ Universidade Tuiuti do Paraná - TUIUTI - Curitiba - Brasil \\ jair.marques@utp.br \\ Rosane Sampaio Santos \\ Universidade Tuiuti do Paraná - TUIUTI - Curitiba - Brasil \\ rosane.santos2@utp.br \\ Kelly Cristina Alves Silvério \\ Faculdade de Odontologia de Bauru da Universidade de São Paulo - FOB-USP-São Paulo - Brasil \\ kcsilver@terra.com.br \\ Eliane Cristina Pereira \\ Universidade Estadual do Centro Oeste - UNICENTRO - Irati- Brasil \\ elianecp@visaonet.com.br \\ Ana Paula Dassie-Leite \\ Universidade Estadual do Centro Oeste - UNICENTRO - Irati- Brasil \\ pauladassie@hotmail.com
}

\section{RESUMO}

OBJETIVO: Investigar a alimentação de idosos com diferentes condições dentárias.

MÉTODOS: Estudo observacional, transversal e descritivo, com 56 idosos (60 a 85 anos), residentes na Região Centro Sul do Estado do Paraná. Os idosos foram divididos em quatro grupos de acordo com o seu estado dentário sendo: idosos com uso de prótese total (G1), uso de prótese parcial (G2), dentição natural (G3) e edentado total (G4). O estado nutricional dos idosos foi determinado através da avaliação antropométrica e da avaliação da ingestão dietética usando o método recordatório 24 horas, com utilização do índice de alimentação saudável adaptado (IASad).

RESULTADOS: Os idosos do G4 (46,7\%) apresentaram baixo peso e valores para Circunferência da Cintura (CC) e Relação Cintura Quadril (RCQ) dentro da normalidade. O G1 $(68,8 \%$ ) e G3 (66,7\%) apresentaram sobrepeso e aumento da CC e RCQ. O G2 (50\%) mostrou-se eutrófico, porém com aumento da CC e da RCQ. De acordo com a classificação do IASad, os idosos do G4 tinham dieta de 'má qualidade' e G1, G2 e G3 tinham dieta 'precisando de melhorias'. Idosos com dieta de 'má qualidade', relataram consumir alimentos na consistência pastosa, líquida e líquida engrossada. Já idosos com dieta 'precisando de melhorias' referiram consumo de alimentos em todas as consistências e apresentavam próteses dentárias firmes na cavidade oral, demonstrando a interferência da condição dentária na escolha dos alimentos. 
CONCLUSÃO: O estado dentário possivelmente foi fator determinante para as escolhas alimentares, a qual refletiu na classificação do IASad e estado nutricional dos idosos.

PALAVRAS-CHAVE: Idosos. Avaliação Nutricional. Saúde Bucal.

\begin{abstract}
OBJECTIVE: investigate the diet in the elderly with different dental conditions.

METHOD: Observational, cross-sectional and descriptive study of 56 seniors (60 to 85 years of age) residents of south central region of the State of Paraná. Subjects were divided into four groups according to their dental status: elderly with total dental prosthesis (G1), with partial prosthesis (G2), natural dentition (G3) and total edentulous (G4). The nutritional status of the elderly was determined by anthropometric measurements and assessment of dietary intake by applying the 24 hour recall method using the Adapted Healthy Eating Index (IASad).

RESULTS: G4 elderly (46.7\%) were underweight and values for Waist circumference (WC) and waist to hip ratio (WHR) within normal range, G1 (68.8\%) and G3 (66.7\%) showed overweight and increased WC and WHR. G2 (50\%) were eutrophic, even though with increased WC and WHR. According with the IASad classification, elderly G4 had a 'poor quality' diet and elderly G1, G2 and G3 had an 'in need of improvement' diet. And had low intakes of calcium, iron and vitamin C. Elderly with a 'poor quality' diet reported food consumption in puree, liquid and thickened liquid consistencies. And elderly with an 'in need of improvement' diet reported food consumption in all consistencies, and dentures presented firmly in the oral cavity, showing the interference of dental condition in food choice.
\end{abstract}

CONCLUSION: Dental status was possibly the determining factor in food choices, which reflected the IASad classification and nutritional status of the elderly.

KEYWORDS: Elderly. Nutrition Assessment. Oral Health.

\title{
1. Introdução
}

Envelhecer é uma realidade na maioria das sociedades desenvolvidas e em desenvolvimento, acarretando desafiospara as políticas públicas, pela necessidade de mudanças de comportamentos e de ações (BRASIL, 2006; MORAIS; RODRIGUES; GERHARDT, 2008).

Desta forma, a busca em proporcionar condições que favoreçam ao idoso uma melhor qualidade de vida está relacionada à ingestão de bons nutrientes. Para que isso aconteça é necessária a presença de dentes naturais sadios ou de próteses dentárias bem adaptadas (CARDOS; BUJES, 2010).

Uma saúde bucal comprometida torna-se mais evidente em idosos que acumulam ao longo da vida as consequências de doenças bucais onde, frequentemente, são refletidas em extensas perdas dentárias, xerostomia, uso de próteses e comprometimentos da mastigação (CARDOS; BUJES, 2010).

O envelhecimento produz modificações estruturais e funcionais, tanto interna como externamente. Tais modificações implicam diretamente nos padrões motores da alimentação apresentadas no sistema estomatognático, que é responsável pela respiração, sucção, mastigação, deglutição e fonação (JALES et al., 2005; CARDOS; BUJES, 2010).

A mastigação mostra-se conectada à saúde bucal, precisamente à saúde dos dentes e à disposição destes de forma regular. Assim, quando a mastigação não ocorre adequadamente, os idosos tendem a recusar a ingestão de muitos alimentos, principalmente associados à xerostomia. Além disso, a alteração nesse aspecto pode ocasionar dificuldades alimentares seguidas de tosse, 
engasgos e pigarros constantes, mesmo quando o idoso não está se alimentando (SUZUKI et al., 2006; CARDOS; BUJES, 2010).

As relações entre o estado bucal e o estado nutricional dos idosos têm indicado que as perdas dentárias e o comprometimento da mastigação estão relacionados à seleção de alimentos, refletindo em prejuízos na nutrição desses indivíduos (MARCENES et al., 2003).

O estado nutricional pode ser definido como a condição de saúde de um indivíduo, influenciada pelo consumo, utilização e necessidades de nutrientes. Vários métodos podem ser utilizados em conjunto na avaliação nutricional como a antropometria, inquéritos alimentares, avaliação física e bioquímica. Um único método não é capaz de definir o estado nutricional do indivíduo, mas pode alertar para possíveis variações desse estado e para o risco de morbidade (MACIEL, 2002; RABITO et al., 2008).

Desta forma, vale destacar a importância da manutenção dos dentes ao longo da vida. O processo de alimentação envolve profissionais de diferentes áreas da saúde como a Nutrição, Odontologia e Fonoaudiologia, possibilitando agregar os mais diversos tipos de conhecimentos fragmentados em prol de uma atenção compartilhada pela qual transcenderá os limites multidisciplinares (SAINTRAIN; VIEIRA, 2008; DIAS; CARDOSO, 2009; ANDRADE et al., 2011).

Portanto, conhecer a relação existente entre o estado dentário e as condições alimentares em idosos é importante para compreender as reais necessidades dessa população. Sendo assim, este estudo busca investigar a alimentação dos idosos com diferentes condições dentárias.

\section{Métodos}

Este estudo, observacional, transversal e descritivo, foi aprovado pelo Comitê de Ética em Pesquisa (CEP) da Universidade Tuiuti do Paraná, sob o protocolo $n^{\circ}$. 00063/2008. Todos os participantes do presente estudo assinaram o Termo de Consentimento Livre e Esclarecido.

Participaram deste estudo 56 idosos, destes, 38 eram do sexo feminino e 18 do sexo masculino (60 a 85 anos), residentes na Região Centro Sul do Estado do Paraná, usuários do Sistema Único de Saúde (SUS).

Foram incluídos no presente estudo idosos com idade igual ou superior a 60 anos; ambos os sexos; com manutenção da dentição natural; ausência de dentição total e/ou parcial; utilização de próteses dentária total ou parcial ou sem utilização de próteses dentárias; com alimentação por via oral exclusiva; deambulando e idosos que demonstraram boa comunicação e compreensão de linguagem. Foram excluídos os idosos com diagnóstico médico de doença neurológica; que realizaram cirurgia de cabeça e pescoço; que apresentaram membros superiores e/ou inferiores com edema e idosos com implantes dentários.

Os idosos foram divididos em quatro grupos de acordo com o seu estado dentário: Grupo 1 (G1) - edentados totais, com uso de prótese total; Grupo 2 (G2) - condição mista de dentição, com menos de 11 falhas dentárias inferiores, utilizando prótese total superior e parcial inferior; Grupo 3 (G3) - dentição natural, com mais de 24 dentes naturais, sem falhas nos pares dentários, não utilizando próteses parciais; Grupo 4 (G4) edentados totais, sem uso de próteses dentárias totais ou parciais.

A escolha da amostra foi aleatória e a coleta dos dados ocorreu nas unidades básicas de saúde ou em outros locais de atendimento de saúde frequentados por eles. Todos os idosos encontravam-se aparentemente nutridos, alimentando-se por via oral, deambulando e comunicativos.

A avaliação dos idosos foi composta pela: Avaliação Nutricional Antropométrica e Dietética - Recordatório 24 horas e Índice de Alimentação Saudável Adaptado (IASad), aplicação do questionário de Autoavaliação da Condição de Mastigação e Deglutição e Avaliação do Estado Dentário (LIMA et al., 2009). Para a realização destas avaliações foram considerados: 
a) Avaliação Nutricional antropométrica: foram obtidas as medidas do peso (kg), estatura $(\mathrm{cm})$, circunferência da cintura $(\mathrm{CC})(\mathrm{cm})$, circunferência do quadril $(\mathrm{cm})$, relação cintura/quadril (RCQ) e o índice de massa corporal (IMC), essas medidas foram utilizadas para classificar o estado nutricional dos idosos;

b) Peso: solicitado aos idosos que retirassem o calçado e o maior número de roupas possível. Cada participante foi posicionado no centro da balança, com os braços na lateral do corpo e sem executar nenhum movimento. $\mathrm{O}$ instrumento utilizado para aferição do peso foi uma balança digital da marca WISO, com capacidade de $180 \mathrm{~kg}$ e intervalo de $100 \mathrm{~g}$;

c) Estatura: solicitado aos idosos que retirassem o calçado e adereços da cabeça, posicionando-se de forma mais ereta possível com os pés alinhados sem estender a cabeça. A barra horizontal foi abaixada até que repousasse no topo da cabeça e, assim, realizada a leitura. $\mathrm{O}$ instrumento utilizado foi o estadiômetro portátil da marca WCS Wood Compact;

d) IMC: obtido pela divisão do peso $(\mathrm{kg})$ pelo quadrado da altura $(\mathrm{m})$, os pontos de corte utilizados foram: Baixo-peso $<22 \mathrm{Kg} / \mathrm{m}^{2}$, Eutrófico $22-27 \mathrm{Kg} / \mathrm{m}^{2}$, Sobrepeso $>27$ $\mathrm{Kg} / \mathrm{m}^{2}$ (LIPSCHITZ, 1994);

e) $C C$ : solicitado ao idoso que ficasse em pé, levantando a blusa para obtenção da medida. Essa medida foi verificada entre as costelas inferiores e a crista ilíaca. A leitura foi feita no momento da expiração. Para coleta desses dados foi utilizada a fita antropométrica da marca WISO $2 \mathrm{~m}$, flexível e inelástica. Os pontos de corte utilizados para o sexo masculino foram abaixo de $\geq 102 \mathrm{~cm}$ e para o sexo feminino $\geq 88 \mathrm{~cm}$, os valores acima destas medidas indicam risco para a saúde (WHO, 2003);

f) Circunferência do Quadril: solicitado para que ficasse em pé de forma ereta, a medida foi feita no nível da sínfise púbica, com a fita métrica, flexível e inelástica, circundando o quadril na parte mais saliente entre a cintura e a coxa, com o idoso usando roupas finas. Para coleta desses dados foi utilizada a fita antropométrica da marca WISO $2 \mathrm{~m}$. A RCQ foi obtida dividindo-se o valor numérico da circunferência da cintura pela circunferência do quadril, ambos em $\mathrm{cm}$. Os pontos de corte utilizados para os idosos do sexo masculino foram $>1,0$ e para o sexo feminino $>0,85$, os valores acima destas medidas indicam risco para a saúde (WHO, 1998);

g) Recordatório 24 horas: foram realizadas perguntas sobre todos os alimentos e bebidas consumidos, por meio de 2 aplicações do recordatório 24 horas. O recordatório foi aplicado em dias alternados, sendo um dia da semana e outro referente ao final de semana. As entrevistas foram individuais. O idoso respondeu sobre o horário em que se alimentou em um período de 24 horas que antecederam a entrevista, o horário das suas refeições, quais os alimentos consumidos, modo de preparo do alimento, nome comercial e suas quantidades, estas relatadas em medidas caseiras, quando necessário demonstrado com utensílios ou formas geométricas para melhor visualização das quantidades;

h) IASad: Os dados dietéticos obtidos em medidas caseiras por meio do recordatório 24 horas foram processados por meio do programa de análise nutricional, o Software Dietwin Professional, versão 2008. Os alimentos não disponíveis no software foram acrescentados, de acordo com a Tabela Brasileira de Composição de Alimentos (NÚCLEO DE ESTUDOS E PESQUISAS EM ALIMENTAÇÃO, 2006), Tabela de Composição de Alimentos (IBGE, 1996) e Tabela para Avaliação de Consumo Alimentar em Medidas Caseiras (PINHEIRO et al., 2002). A pontuação de cada componente da dieta variou entre 0 a 10 , totalizando 100 pontos como nota máxima, sendo classificado como uma alimentação de 'boa qualidade' quem obteve mais de 100 pontos, 'precisando de melhorias' de 71 a 100 pontos e uma dieta de 'má qualidade' inferior a 71 pontos (MOTA et al., 2008);

i) Autoavaliação da Condição de Mastigação e Deglutição: Foi aplicado um questionário de percepção da mastigação, adaptado do protocolo de entrevista de Lima et al.(2009), 
com perguntas abertas e fechadas, em que o participante respondeu a um nutricionistapesquisador, que fez perguntas sem interferir nas respostas. Sobre as condições de mastigação, as perguntas contidas no questionário foram: Qual a consistência dos alimentos que você consome? Sendo possíveis as respostas: Sólido, Pastoso, Líquido, Líquido engrossado e Todas as consistências; Quanto ao tipo de alimentos ingeridos, as perguntas foram: Sente dificuldade em mastigar algum alimento? Sendo as possíveis respostas: Sim, Não e solicitado para citar quais alimentos; Sente dor durante a mastigação? Com as possíveis respostas: Sim, Não, Às vezes e questionado o local da dor; Você sente cansaço para mastigar algum alimento? Tendo como respostas: Sim, Não, Às vezes e solicitado para referir quais os alimentos; Você sente dificuldade em mastigar algum alimento que gosta de comer? Com as possíveis respostas: Sim, Não e questionado quais os alimentos; Você mastiga dos dois lados? Com as possíveis respostas: Sim e Não. Em caso da resposta ser negativa, era questionado qual o lado Direito e Esquerdo; Você acha que seu prazer em se alimentar alterou em relação à antigamente? Com as possíveis respostas: Sim e Não; Como você classifica a sua mastigação com a prótese? Com as possíveis respostas: Ótima, Boa, Regular e Ruim; Como você classificaria a sua Deglutição com a Prótese? Com as possíveis respostas: Ótima, Boa, Regular e Ruim;

j) Avaliação do Estado Dentário: Com o auxílio de um profissional odontólogo, foi aplicado um questionário, adaptado de Lima et al.(2009) e do Conselho Federal de Odontologia (2011), com perguntas fechadas e abertas sobre o estado geral da dentição do idoso. A avaliação odontológica aconteceu de acordo com um prévio agendamento, na unidade básica de saúde. O profissional odontólogo é membro da Unidade Básica de Saúde, e avaliou a dentição dos idosos do estudo. Para a coleta destes dados, o idoso ficou sentado em uma cadeira e o odontólogo analisou e avaliou sua dentição, quando necessário respondeu algumas perguntas presentes no questionário. Foram utilizados dois odontogramas, o primeiro para assinalar as falhas dentárias existentes e em quais elementos elas se encontravam, o segundo odontograma foi utilizado para identificar se nessas falhas o indivíduo utilizava alguma prótese dentária total, parcial, pivô, ponte, não utiliza ou se apresentava dentição natural. O estado das próteses e dentição foi classificado como 'bom' estado de conservação, 'regular' estado de conservação e 'mau' estado de conservação (LIMA et al., 2009). As perguntas contidas no questionário eram as seguintes: Há quanto tempo utiliza a prótese? Com as possíveis respostas: menos de 5 anos, de 5 a 10 anos, de 10 a 20 anos, mais de 20 anos; Teve dificuldade na adaptação da prótese? Com as possíveis respostas: Sim e Não; A prótese está firme na boca? Com as possíveis respostas: Sim e Não; A prótese está machucando? Com as possíveis respostas: Sim e Não; Faz acompanhamento periódico com o dentista? Com as possíveis respostas: Sim e Não; Usa prótese para se alimentar? Com as possíveis respostas: Sim e Não; Você usa algum tipo de adesivo para fixar sua prótese? Com as possíveis respostas: Sim e Não; Tem dificuldade para mastigar com a prótese? Com as possíveis respostas: Sim, Não e relatado com quais os alimentos sente a dificuldade para mastigar com a prótese; Você está satisfeito com a condição atual de sua prótese? Com as possíveis respostas:

Sim, Não e quando negativo questionado o motivo.

$\mathrm{Na}$ análise estatística foram utilizados métodos descritivos (média, mínimo, máximo, desvio padrão e arranjos tabulares de acordo com as frequências absolutas e relativas).

\section{Resultados}

Seguem os dados encontrados sobre o IMC, a CC e a RCQ dos idosos de acordo com a classificação do estado dentário: 
Tabela 1 - Classificação dos idosos por grupos de acordo com a avaliação Antropométrica (n=56)

\begin{tabular}{|c|c|c|c|c|c|c|}
\hline \multicolumn{2}{|c|}{ Classificações } & \multicolumn{4}{|c|}{ Grupos } & \multirow{2}{*}{$\begin{array}{c}\text { Amostra } \\
\text { Total }\end{array}$} \\
\hline & & Grupo 1 & Grupo 2 & Grupo 3 & Grupo 4 & \\
\hline \multicolumn{7}{|c|}{ Indice De Massa Corporal $\left(\mathrm{kg} / \mathrm{m}^{2}\right)$} \\
\hline Baixo peso & $<22$ & $1(6,2 \%)$ & $2(12,5 \%)$ & $-(0,0 \%$ & $7(46,7 \%)$ & 10 \\
\hline Eutrófico & $22-27$ & $4(25,0 \%)$ & $8(50,0 \%)$ & $3(33,3 \%)$ & $3(20,0 \%)$ & 18 \\
\hline Sobrepeso & $>27$ & $11(68,8 \%)$ & $6(37,5 \%)$ & $6(66,7 \%)$ & $5(33,3 \%)$ & 28 \\
\hline \multicolumn{7}{|c|}{ Circunferência da Cintura } \\
\hline \multirow[t]{2}{*}{ Masculino } & $\geq 102 \mathrm{~cm}$ & $3(18,8 \%)$ & $2(12,5 \%)$ & $1(11,1 \%)$ & $4(26,7 \%)$ & 10 \\
\hline & $<102 \mathrm{~cm}$ & $1(6,2 \%)$ & $1(6,2 \%)$ & $-(0,0 \%)$ & $6(40,0 \%)$ & 8 \\
\hline \multirow[t]{2}{*}{ Feminino } & $\geq 88 \mathrm{~cm}$ & $11(68,8 \%)$ & $9(56,3 \%)$ & $8(88,9 \%)$ & $3(20,0 \%)$ & 31 \\
\hline & $<88 \mathrm{~cm}$ & $1(6,2 \%)$ & $4(25,0 \%)$ & $-(0,0 \%)$ & $2(13,3 \%)$ & 7 \\
\hline \multicolumn{7}{|c|}{ Relação Cintura/Quadril } \\
\hline \multirow[t]{2}{*}{ Masculino } & $>1,0$ & $2(12,5 \%)$ & $1(6,2 \%)$ & $1(11,1 \%)$ & $5(33,3 \%)$ & 9 \\
\hline & $\leq 1,0$ & $2(12,5 \%)$ & $2(12,5 \%)$ & $-(0,0 \%)$ & $5(33,3 \%)$ & 9 \\
\hline \multirow[t]{2}{*}{ Feminino } & $>0,85$ & $11(68,8 \%)$ & $7(43,8 \%)$ & $6(66,7 \%)$ & $3(20,0 \%)$ & 27 \\
\hline & $\leq 0,85$ & $1(6,2 \%)$ & $6(37,5 \%)$ & $2(22,2 \%)$ & $2(13,3 \%)$ & 11 \\
\hline TOTAL & & 16 & 16 & 9 & 15 & 56 \\
\hline
\end{tabular}

Fonte: Autoria própria (2012).

Legenda: Grupo 1 - edentados totais, com uso de prótese total; Grupo 2 - condição mista de dentição, com menos de onze falhas dentárias inferiores, utilizando prótese total superior e parcial inferior; Grupo 3 - dentição natural, com mais de 24 dentes naturais, sem falhas nos pares dentários, não utilizando próteses parciais; Grupo 4 edentados totais, sem uso de próteses dentárias totais ou parciais.

A seguir, a distribuição dos idosos de acordo com a classificação do estado dentário e a classificação da qualidade da alimentação segundo o IASad:

Tabela 2 - Distribuição da amostra por grupos de acordo com a classificação do IASad

\begin{tabular}{l|c|c|c|c|c}
\hline \multirow{2}{*}{ Classificação do IASad } & \multicolumn{5}{|c}{ Grupos } \\
\cline { 2 - 6 } & Grupo 1 & Grupo 2 & Grupo 3 & Grupo 4 & $\begin{array}{c}\text { Amostra } \\
\text { Total }\end{array}$ \\
\hline Boa qualidade & $1(6,2 \%)$ & $6(37,5 \%)$ & $3(33,3 \%)$ & - & 10 \\
Má qualidade & - & $2(12,5 \%)$ & - & $8(53,3 \%)$ & 10 \\
Precisam melhorias & $15(93,8 \%)$ & $8(50,0 \%)$ & $6(66,7 \%)$ & $7(46,7 \%)$ & 36 \\
\hline TOTAL & 16 & 16 & 9 & 15 & 56 \\
\hline
\end{tabular}

Fonte: Autoria própria (2012).

Legenda: Grupo 1 - edentados totais, com uso de prótese total; Grupo 2 - condição mista de dentição, com menos de onze falhas dentárias inferiores, utilizando prótese total superior e parcial inferior; Grupo 3 dentição natural, com mais de 24 dentes naturais, sem falhas nos pares dentários, não utilizando próteses parciais; Grupo 4 edentados totais, sem uso de próteses dentárias totais ou parciais.

A distribuição dos idosos de acordo com a classificação da qualidade da alimentação e as consistências alimentres ingeridas estão descritas na tabela seguinte:

Tabela 3 - Relação entre o índice de alimentação saudável adaptado e aconsistência dos alimentos

\begin{tabular}{|c|c|c|c|c|c|c|c|}
\hline \multirow[b]{2}{*}{$\begin{array}{c}\text { Classificação } \\
\text { do IASad }\end{array}$} & \multicolumn{6}{|c|}{ CONSISTÊNCIA DOS ALIMENTOS } & \multirow[b]{2}{*}{ Total } \\
\hline & Todas & Sólido & Pastoso & $\begin{array}{l}\text { Pastoso/ } \\
\text { Sólido }\end{array}$ & $\begin{array}{c}\text { Pastoso/ } \\
\text { Líquido/ } \\
\text { Líquido } \\
\text { engrossado }\end{array}$ & $\begin{array}{c}\text { Pastoso/ } \\
\text { Sólido/ } \\
\text { Líquido/ } \\
\text { Líquido } \\
\text { engrossado }\end{array}$ & \\
\hline Boa qualidade & $7(21,9 \%)$ & - & - & - & $2(11,8 \%)$ & $1(100 \%)$ & 10 \\
\hline Má qualidade & - & - & $2(100 \%)$ & - & $8(47,1 \%)$ & - & 10 \\
\hline $\begin{array}{l}\text { Precisam } \\
\text { melhorias }\end{array}$ & $25(78,1 \%)$ & $1(100 \%)$ & - & $3(100 \%)$ & $7(41,1 \%)$ & - & 36 \\
\hline TOTAL & 32 & 1 & 2 & 3 & 17 & 1 & 56 \\
\hline
\end{tabular}

Fonte: Autoria própria (2012). 
A seguir a distribuição dos idosos de acordo com a consistência alimentar ingerida e a firmeza da prótese dentária:

Tabela 4 - Relação entre consistência dos alimentos e se a prótese permanecefirme na cavidade oral

\begin{tabular}{|c|c|c|c|c|c|c|c|}
\hline \multirow[b]{2}{*}{$\begin{array}{c}\text { Prótese } \\
\text { Firme }\end{array}$} & \multicolumn{6}{|c|}{ Consistência dos Alimentos } & \multirow[b]{2}{*}{ Total } \\
\hline & Todas & Sólido & Pastoso & $\begin{array}{c}\text { Pastoso/ } \\
\text { Sólido }\end{array}$ & $\begin{array}{c}\text { Pastoso/ } \\
\text { Líquido/ } \\
\text { Líquido } \\
\text { engrossado }\end{array}$ & $\begin{array}{c}\text { Pastoso/ } \\
\text { Sólido/ } \\
\text { Líquido/ } \\
\text { Líquido } \\
\text { engrossado }\end{array}$ & \\
\hline Não & $6(18,8 \%)$ & - & $1(50 \%)$ & $1(33,3 \%)$ & $2(11,8 \%)$ & $1(100 \%)$ & 11 \\
\hline Sim & $14(43,7 \%)$ & $1(100 \%)$ & $1(50 \%)$ & $1(33,3 \%)$ & $1(5,9 \%)$ & - & 18 \\
\hline $\begin{array}{l}\text { Superior sim } \\
\text { e inferior } \\
\text { não }\end{array}$ & $4(12,5 \%)$ & - & - & $1(33,3 \%)$ & $1(5,9 \%)$ & - & 6 \\
\hline Não usa & $8(25,0 \%)$ & - & - & - & $13(76,4 \%)$ & - & 21 \\
\hline TOTAL & 32 & 1 & 2 & 3 & 17 & 1 & 56 \\
\hline
\end{tabular}

Fonte: Autoria própria (2012).

\section{Discussão}

Ao envelhecer as perdas dentárias tornam-se marcantes. Além de comprometer a estética, a fala, a mastigação, a deglutição e estado nutricional, esse fato passa a ser motivo de preocupação para a saúde geral desses indivíduos (CARDOS; BUJES, 2010).

Além disso, as perdas dentárias afetam a qualidade dietética e o aporte nutricional de tal forma que podem aumentar o risco para diversas doenças sistêmicas, contribuindo para mudanças de peso (MARCHI et al., 2008).

Uma boa saúde bucal durante toda a vida e na velhice tem mostrado influência na qualidade de vida dos idosos, pois proporciona sensação de bem-estar e positiva autoestima, melhora a mastigação, a ingestão de alimentos, a comunicação e reduz o número de doenças vinculadas ao processo saúde-doença-cuidado (MARSHALL et al.,2002).

Com relação à avaliação antropométrica que objetivou classificar o estado nutricional dos idosos, os valores obtidos para o IMC no grupo dos edentados totais (G4) evidenciou maior ocorrência de baixo peso. O que pode significar que ser edentado e não usar qualquer tipo de prótese é um fator relevante e propicia maiores índices de baixo peso. Já os idosos com uso de prótese dentária total e com dentição natural (G1 e G3) apresentaram maior ocorrência de sobrepeso. Provavelmente, o sobrepeso evidenciado nesses idosos apresente relação com o próprio processo de envelhecimento, pois um bom estado nutricional somente é mantido quando há equilíbrio entre ingestão alimentar e gasto energético.

Os valores do IMC e de massa corporal aumentam com o avanço da idade, os hábitos alimentares inadequados e o sedentarismo são os principais aspectos que levam a um desequilíbrio nessa relação (SANTOS; SICHIERI, 2005; NESPECA; CYRILLO, 2010).

Dados semelhantes aos encontrados no presente estudo também foram obtidos por Sheiham et al. (2002), ao avaliarem 629 idosos ingleses, evidenciando que idosos edentados apresentam mais baixo-peso (IMC $<20 \mathrm{Kg} / \mathrm{m} 2)$ que aqueles com 10 ou mais dentes.

Analisando uma população com as mesmas características, Marcenes et al. (2003) verificaram que idosos edentados apresentaram mais baixo peso do que aqueles com 11 ou mais dentes. No entanto, tais autores utilizaram outros pontos de corte para a classificação do IMC e a idade mínima foi de 65 anos, enquanto no presente estudo foi de 60 anos.

Ainda de acordo com os dados antropométricos, os valores da $\mathrm{CC}$ dos idosos com uso de prótese total, parcial e dentição natural (G1, G2, G3) do sexo feminino apresentaram-se aumentados, o que demonstrou predomínio de idosos com acúmulo de gordura abdominal. Os idosos edentados (G4) apresentaram maior ocorrência de valores normais para essa medida devido ao baixo peso apresentado por esse grupo. 
Tais dados também foram encontrados por Félix e Souza (2009) quanto ao aumento da CC que esteve presente em $86,4 \%$ das mulheres e em $57,1 \%$ dos homens. Em outro estudo, Santos e Sichieri (2005) também observaram maior proporção de inadequação entre as mulheres.

De acordo com a avaliação da RCQ, a proporção de inadequação dessa medida foi encontrada com maior ocorrência nos idosos do sexo feminino, com uso de prótese total, parcial e dentição natural (G1, G2 e G3), quando comparado ao sexo masculino. O aumento da RCQ esteve presente apenas nos idosos edentados (G4) do sexo masculino.

Esses dados foram encontrados por vários estudos, evidenciando que idosos do sexo feminino apresentaram uma RCQ mais inadequada quando comparados ao sexo masculino, pois com o aumento da idade há uma modificação na gordura corporal, diminuição dos tecidos gordurosos dos braços e das pernas e aumento do abdômen (SAMPAIO, 2004; TINOCO et al.,2006).

Em outro estudo, Santos e Sichieri (2005) verificaram que as idosas apresentaram maior proporção de inadequação da RCQ com relação aos idosos. Félix e Souza (2009) verificaram que a RCQ, quando acima do recomendado, além de evidenciar alta proporção de risco cardiovascular em ambos os sexos, aponta maior risco entre as mulheres $(95,5 \%)$ quando comparado aos homens $(42,9 \%)$.

Com relação à qualidade alimentar, Volp et al. (2010) verificaram que a utilização do IASad permite estudar a qualidade total da dieta de idosos, e não somente de componentes isolados, observando que quanto maior o escore do IASad, mais é aumentada a ingestão de nutrientes.

Na presente pesquisa, 93,7\% dos idosos com prótese total (G1), 50\% parcial (G2) e 66,6\% com dentição natural (G3) apresentaram uma alimentação, de acordo com o IASad, como 'precisando de melhorias' e 53,3\% dos idosos edentados totais (G4) apresentaram uma alimentação de 'má qualidade'. Tais classificações são reflexos das escolhas alimentares, dessa forma, o estado dentário provavelmente interferiu nessas escolhas.

Dados semelhantes foram obtidos por Malta, Papini e Corrente (2013), com idosos integrados ao Programa Saúde da Família e Unidades Básicas de Saúde do Município de Avaré, São Paulo. Os autores verificaram que 32,9\% dos idosos apresentaram dieta de 'má qualidade', 60,3\% 'precisam de melhorias' e apenas 6,8\% apresentaram uma dieta de 'boa qualidade'.

Ainda de acordo com o mesmo estudo, a classificação do IASad foi reflexo do número médio de porções de frutas, hortaliças, cereais, leite e derivados ingeridos, que ficaram abaixo do mínimo recomendado em relação aos componentes leguminosas, carnes e ovos, açúcar e óleo, onde o consumo ultrapassou a média.

De acordo com os dados do presente estudo, verificou-se que os idosos que referiram ingerir alimentos de todas as consistências relataram que a prótese dentária permanece firme durante a mastigação dos alimentos, porém, apresentaram uma dieta 'precisando de melhorias', e os que referiram ingerir uma dieta com consistência líquida, líquida engrossada e/ou pastosa, devido ao seu estado dentário, evidenciaram uma dieta de 'má qualidade'. Isso indica que o estado dentário atual dos idosos, reflete nas escolhas alimentares, consistência dos alimentos e classificação do IASad.

A dificuldade para mastigar, de acordo com o estudo realizado por Fazito, Perim e Di Ninno (2004), está mais evidenciada nos idosos edentados (61,9\%), quando comparada aos dentados $(38,1 \%)$. Em outro estudo, Lima et al (2009) observaram que o mau estado de conservação dos dentes e próteses dificulta o processo mastigatório comprometendo a ingestão de nutrientes.

Entre os idosos de baixa renda do Reino Unido, 21,9\% dentados e 36,3\% edentados apresentaram dificuldades em mastigar algum alimento, pois ser edentado contribui de forma negativa para a capacidade mastigatória, em relação ao consumo de uma variedade de alimentos (TSAKOS et al., 2010).

Idosos edentados desenvolvem o hábito de amassar os alimentos com a língua pressionandoos contra o palato, devido à impossibilidade de ciclos oclusais, deixando dessa forma a deglutição prejudicada (CARDOS; BUJES, 2010).

Com o avanço da idade ocorrem mudanças nas estruturas e funções miofuncionais e orofaciais, levando o idoso a habituar-se às dificuldades que ocorrem no processo de deglutição. 
Esses fatores podem estar relacionados às falhas dentárias, ao uso de próteses mal adaptadas e mudanças musculares, em que a ingestão de líquidos torna-se necessária como meio facilitador quando se ingere alimentos sólidos (DIAS; CARDOSO, 2009), e até mesmo uma mudança na consistência alimentar pode ser necessária.

\section{Considerações finais}

Pelo próprio processo de envelhecimento, os idosos, em geral, tendem a apresentar dietas comprometidas. A ausência total de dentes pode influenciar nas escolhas alimentares e consequentemente o estado nutricional, acarretando em baixo peso. Considerando a complexidade desses fatores, há necessidade de uma crescente consciência por parte dos profissionais de saúde a respeito da importância de manter a saúde bucal com a finalidade de atingir um bom estado nutricional entre os idosos.

Foi possível verificar relação entre o estado dentário, índice de alimentação saudável, estado nutricional e condição de mastigação entre os idosos, visto que a utilização da prótese dentária bem adaptada parece minimizar os prejuízos alimentares e nutricionais nos idosos estudados. Desta forma, o estado dentário possivelmente foi o fator determinante para as escolhas alimentares, a qual refletiu na classificação do IASad e estado nutricional.

Esses conhecimentos devem ser discutidos e direcionados de forma interdisciplinar, pois são essenciais na assistência ao idoso, sendo que as ações devem ser compartilhadas em diversos saberes de acordo com a realidade de cada indivíduo. Novas pesquisas são necessárias para propiciar maior conhecimento nessa área e, assim, estabelecer práticas de monitoramento e intervenções mais direcionadas às necessidades da população idosa.

\section{Referências}

ANDRADE, F. B. et al. The relationship between nutrient intake, dental status and family cohesion among older Brazilians. Caderno de Saúde Pública, v. 27, n. 1, p. 113-122, jan. 2011.

BRASIL. Ministério da Saúde. Secretaria de Atenção à Saúde. Departamento de Atenção Básica. Caderno de Atenção Básica - Envelhecimento e Saúde da Pessoa Idosa. Brasília, 2006.

CARDOS, M. C. A. F.; BUJES, R. V. A saúde bucal e as funções da mastigação e deglutição nos idosos. Estudos Interdisciplinares sobre o Envelhecimento, v. 15, n. 1, p. 53-67, 2010.

CONSELHO FEDERAL DE ODONTOLOGIA. ODONTOGRAMA. Disponível em: <http://cfo.org.br/wp-content/uploads/2009/10/odontograma_exame.pdf>. Acesso em: 16 jun. 2011.

DIAS, B. K. P.; CARDOSO, M. C. A. F. Características da função de deglutição em um grupo de idosas institucionalizadas. Estudos Interdisciplinares sobre o Envelhecimento, v. 14, n. 1, p. 107$124,2009$.

FAZITO, L. T.; PERIM, J. V.; DI NINNO, C. Q. M. S. Comparação das queixas alimentares de idosos com e sem prótese dentária. Revista CEFAC, v. 6, n. 2, p. 143-150, abr./jun. 2004.

FÉLIX, L. N.; SOUZA, E. M. T. Avaliação nutricional de idosos em uma instituição por diferentes instrumentos. Revista de Nutrição, v. 22, n. 4, p. 571-580, ago. 2009.

INSTITUTO BRASILEIRO DE GEOGRAFIA E ESTATÍSTICA - IBGE. Tabela de Composição de Alimentos. 4. ed. Rio de Janeiro: IBGE, 1996. 
JALES, M. A. et al. Características do Sistema Estomatognático em Idosos: Diferenças entre instituição Pública e Privada. Revista CEFAC, v. 7, n. 2, p. 178-187, 2005.

LIMA, R. M. F. et al. Adaptações na mastigação, deglutição e fonoarticulação em idosos de instituição de longa permanência. Revista CEFAC, v. 11, n. 3, p. 405-422, 2009.

LIPSCHITZ, D. A. Screening for nutritional status in the elderly. Nutrition in Old Age, v. 21, n. 1, p. 55-67, mar. 1994.

MACIEL, A. Avaliação multidisciplinar do paciente geriátrico. Rio de Janeiro: Revinter, 2002.

MALTA, M. B.; PAPINI, J. S.; CORRENTE, J. E. Avaliação da alimentação de idosos de município paulista - aplicação do Índice de Alimentação Saudável. Revista Ciência e Saúde Coletiva, v. 18, n. 2, p. 377-384, 2013.

MARCENES, W. et al. The relationship between dental status, food selection, nutrient intake, nutritional status, and body mass index in older people. Caderno de Saúde Pública, v. 19, n. 3, p. 809-815, 2003.

MARCHI, R. J. et al. Association between oral health status and nutritional status in south Brazilian independent-living older people. Nutrition, v. 24, p. 546-553, 2008.

MARSHALL, T. A. et al. Oral health, nutrient intake and dietary quality in the very old. JADA, v. 133, n. 10, p. 1369-1379, Oct. 2002.

MORAIS, E. P.; RODRIGUES, R. A. P.; GERHARDT, T. E. Os idosos mais velhos no meio rural: realidade de vida e saúde de uma população do interior gaúcho. Texto contexto - Enfermagem, v. 17, n. 2, p. 374-383, jun. 2008.

MOTA, J. F. et al. Adaptação do índice de alimentação saudável ao guia alimentar da população brasileira. Revista Nutrição, v. 21, n. 5, p. 545-552, out. 2008.

NESPECA, M.; CYRILlO, D. C. Avaliação da qualidade da dieta por meio do índice de alimentação saudável de funcionários de uma universidade pública. Nutrire: Revista Sociedade Brasileira de Alimentação e Nutrição, v. 35, n. 2, p. 81-90, ago. 2010.

NÚCLEO DE ESTUDOS E PESQUISA EM ALIMENTAÇÃO. Universidade Estadual de Campinas. Tabela brasileira de composição de alimentos. Versão II. 2. ed. Campinas: Unicamp, 2006.

PINHEIRO, A. B. V. et al. Tabela para avaliação de consumo alimentar em medidas caseiras. 5. ed. São Paulo: Atheneu, 2002.

RABITO, E. I. et al. Validation of predictive equations for weight and height using a metric tape. Nutricion Hospitalaria, v. 23, n. 6, p. 614-618, 2008.

SAINTRAIN, M. V. L.; VIEIRA, L. J. E. S. Saúde bucal do idoso: abordagem interdisciplinar. Ciência e Saúde Coletiva, v. 13, n. 4, p. 1127-1132, jul./ago. 2008.

SAMPAIO, L. R. Avaliação nutricional e envelhecimento. Revista de Nutrição, Campinas, v. 17, n. 4, p.507-514, out./dez. 2004. 
SANTOS, D. M.; SICHIERI, R. Índice de massa corporal e indicadores antropométricos de adiposidade em idosos. Revista de Saúde Pública, v. 39, n. 2, p. 163-168, abr. 2005.

SHEIHAM, A. et al. The relationship between oral health status and Body Mass Index among older people: a national survey of older people in Great Britain. British Dental Journal, v. 192, n. 12, p. 703-706, jun. 2002.

SUZUKI, H. S. et al. Avaliação clínica e videofluoroscópica de pacientes com distúrbios da deglutição - estudo comparativo em dois grupos etários: adultos e idosos. Arquivos de Gastroenterologia, v. 43, n. 3, p. 201-205, jul./set. 2006.

TINOCO, A. L. A. et al. Sobrepeso e obesidade medidos pelo índice de massa corporal (IMC), circunferência da cintura (CC) e relação cintura/quadril (RCQ), de idosos de um município da Zona da Mata Mineira. Revista Brasileira de Geriatria e Gerontologia, v. 9, n. 2, p. 63-73, 2006.

TSAKOS, G. et al. Edentulism and Fruit and Vegetable Intake in Low-income Adults. Journal of Dental Research, v. 89, n. 5, p. 462-467, 2010.

VOLP, A. C. P. et al. Índices dietéticos para avaliação da qualidade de dietas. Revista de Nutrição, v. 23, n. 2, p. 281-296, abr. 2010.

WORLD HEALTH ORGANIZATION. Obesity: preventing and managing the global epidemic. Report of a WHO Consulation on Obesity. Geneva. 1998.

WORLD HEALTH ORGANIZATION. Recomendaciones para la prevención del aumento excesivo de peso y la obesidad. Serie de Informes Técnicos 916. Geneva. 2003. 